\title{
Racial and Ethnic Differences in Breast Cancer Patients: A Study for Michigan and California
}

\author{
Hafiz MR Khan ${ }^{1 *}$, Anshul Saxena ${ }^{2}$, Aamrin Rafiq ${ }^{3}$, Sarah Mende ${ }^{4}$, Summre Blakely ${ }^{1}$, \\ Maria Lyuksyutova ${ }^{4}$, and Samantha Jayasinghe ${ }^{4}$ \\ ${ }^{1}$ Department of Public Health, Texas Tech University Health Sciences Center, Lubbock, Texas 79430, USA \\ ${ }^{2}$ Department of Health Promotion and Disease Prevention, Florida International University, Miami, FL 33199, USA \\ ${ }^{3}$ Department of Computer Science, Texas Tech University, Lubbock, Texas 70409, USA \\ ${ }^{4}$ School of Medicine, Texas Tech University Health Sciences Center, Lubbock, Texas 79430, USA
}

Received: 07 August, 2017; Accepted: 14 August, 2017, 2017; Published: 25 September, 2017

*Corresponding author: Hafiz MR Khan, Department of Public Health, Texas Tech University Health Sciences Center, Lubbock, Texas 79430, USA, Tel: 806-743-4213; E-mail: hafiz.khan@ttuhsc.edu

\begin{abstract}
Background: Breast cancer is a leading cause of cancer death for women. Disparities in patient survival should be investigated to improve treatment and prevention methods. The SEER cancer registries have collected most of the breast cancer data for Michigan and California compared to other states. It is important to discern whether there are racial and ethnic differences in age of diagnosis and survival times for the breast cancer patients. Therefore, this study examines variations in age of diagnosis and survival time for breast cancer patients in California and Michigan for four ethnic populations: White Hispanics, White non-Hispanics, Black Hispanics, and Black non-Hispanics.
\end{abstract}

Methods: This study used SEER data from 1973-2012 with 1,000 randomly selected subjects from each ethnic group (except Black Hispanics). Patient data were analyzed for each state based on the mean age of diagnosis and mean survival time. The Cox Proportion Hazard Ratio method and a goodness-of-fit assessment compared survival probability between both states. A cross-tabulation analysis compared different age categories and race.

Results: The White Hispanic population in California had a significantly higher mean age of diagnosis than White Hispanics in Michigan (59.2 \pm 14.6 versus $56.9 \pm 13.9$ years; $p<0.001$ ). Survival times for White non-Hispanics, Black non-Hispanics, and Black Hispanics in California were significantly higher $(p<0.05)$ than survival times for those ethnic groups in Michigan. The goodnessof-fit probability test contributed two main probability distributions, t-distribution and Chi-squared distribution, that showed the best fit among the various ethnic groups in both states.

Conclusion: Racial disparities exist within survival time and age of diagnosis statistics of breast cancer patients in California and Michigan. These findings can be used by healthcare professionals to create population-specific prevention methods to reduce racial and ethnic disparities among breast cancer patients.

Keywords: Breast cancer data; statistical probability models; goodness-of-fit tests

\section{Introduction}

Breast cancer is the most commonly diagnosed cancer in women in the U.S. and is a serious public health issue that needs to be addressed. Breast cancer affected an estimated 246,660 lives in 2016 alone, with an estimated 30\% of newly-diagnosed cancer cases in the U.S. to have been breast cancer diagnoses [1,2,3]. An additional 40,610 female deaths are expected in 2017 due to breast cancer [4]. Breast cancer has a widespread impact, and places a significant financial burden on the population. In 2010, the prevalence costs of cancer care in the United States were totaled around $\$ 124.5$ billion, with breast cancer draining the most money of all other cancers at $\$ 16.5$ billion [4]. By 2020 , this cost is estimated to increase to $\$ 20.2$ billion. Better understanding of the breast cancer patient population in the U.S. could reduce costs through prevention.

There are disparities in survival and diagnosis statistics between different races and ethnicities among breast cancer patients. Compared to African Americans in the United States, Caucasians have a much higher incidence of breast cancer among women aged 40 or older [1,2]. Caucasians are shown to also have much higher survival rates and better overall prognosis compared to African Americans. African Americans, Hispanic Whites, and American Indians have poorer disease-specific survival rates and have a higher incidence of being diagnosed with hormone receptor negative tumors [5,6]. Although African Americans have a lower incidence of breast cancer compared to other races, they typically have a higher incidence of more aggressive subtypes of breast cancer, such as triple-negative breast cancer. More aggressive subtypes are typically classified by tumor expression of Estrogen Receptors (ER), Progesterone Receptors (PR), and Human Epidermal Growth Factor Receptor 2 (HER2) [7]. ER tumors are classified as either ER-positive or ER-negative, with ER-positive tumors having a marginally better survival rate due 
to a response to endocrine therapy [8]. Estrogen essentially fuels a growing breast cancer; thus the presence of estrogen receptors is a good indicator of a more aggressive tumor. The absence of PR expression in ER - positive tumors may scramble typical growth signaling which contributes to tamoxifen resistance [9]. HER2positive tumors have been associated with increased recurrence rates, occurrence in younger women, higher nuclear grade suggesting increased tumor aggressiveness, positive margins during tissue excision (cancer cells extending beyond the area of tissue removed), and increased mortality [10]. The study of survival time differences among different racial and ethnic populations of breast cancer patients could reveal information for clinicians to better address breast cancer.

Hispanic Americans also have high incidence rates of specific cancers compared to Caucasians. Despite this known increased incidence in this group, there have been limitations in their involvement in cancer prevention programs [11]. Compared to other ethnicities, Hispanic American and African American women are less likely to undergo breast cancer screening [12]. This could be due to cultural beliefs and decreased awareness or education of cancer risk factors, which can be influential on breast cancer screening [13]. A lack of screening can cause a patient to be diagnosed at a later stage of cancer, leading to a decreased survival time and a less optimistic treatment plan [14]. Furthermore, several psychological barriers exist, such as fear of a cancer diagnosis as well as fear of pain with mammogram procedures, which may play a role in limiting breast cancer prevention programs in the Hispanic female population [15]. In order to have a culturally appropriate response to the Hispanic population, it is important for physicians and public health officials to consider the importance of cultural competence in prevention methods.

Other potential factors influencing a patient's survival time and time of diagnosis include low socioeconomic status, a reduced access to healthcare, and a lower frequency of mammography and screening [14]. Some studies have also shown a link between an increased Body Mass Index (BMI) and high-grade cancers [16]. Further investigation could determine if unidentified environmental exposures or genetic differences are causing racial disparities in breast cancer incidence and survival times. In order to ease these high rates of incidence and increase patients' survival time, preventive programs should be targeted to high-risk populations for breast cancer. In 2009, the United States Preventive Services Task Force (USPSTF) focused on mammography screening for women ages 50 and older and stated in a report that "women ages 40-49 attain less benefit with more risk" from mammography screening [17]. This was due to the lack of additional research to study the benefits of early mammography screening, as well as the fact that mammograms were not yet fully covered by all insurance companies [17]. In recent years, more emphasis has been placed on earlier mammography screenings. Emphasis on earlier mammography screening was reinforced with the 2016 legislature from the Affordable Care Act (ACA) that ensures most all insurance companies fully cover mammograms in women age 40 and over with no cost sharing [17]. A recent study examined the effects of the USPSTF public announcement on screening rates for breast cancer, of which a small reduction was found [18]. Similar methods could be used as tools for the future to increase awareness and screening rates in high-risk populations, and indirectly improve survival times in women of all races and ethnicities [18]. Extensive study in the differences between survival of breast cancer patients among various states and ethnicities can be found in the works of Khan, et al. [1922]. These studies have thorough analyses of the SEER data to investigate the geographic, socioeconomic, and racial effects on breast cancer patients and their treatment and survival. These populations should be further investigated to discover possible reasons for higher incidence rates and other possible methods to prevent them.

The present study considers breast cancer patient data from two representative states, Michigan and California because these two states were chosen to represent discrete regions in the United States. Michigan provides a data sample representative of the breast cancer population in northern region of the U.S., and California provides a data sample for western region. The majority of the data from the SEER cancer registries is found for Michigan and California, therefore, this study considers only those two states.

A study of Michigan breast cancer survival rates from the years 1985 - 2002 from the Michigan Cancer Surveillance Program found that White non-Hispanics had significantly better survival rates compared to African Americans throughout southern Michigan. However, when Michigan was divided into smaller geographic areas, the differences in breast cancer survival rates were no longer evident. Smaller geographic areas could be more cohesive with access to screening facilities and healthcare. This cohesion could explain the lack of racial disparity with breast cancer outcomes in smaller geographic areas, and presents the idea that breast cancer survival rates may not be due to racial disparity but regional disparity in Michigan [23]. Another study examined the breast cancer outcomes of Michigan by race, socio-economic status, incidence, stage at diagnosis, screening, mortality, and survival among women ages 50 years and older from 1987-2005. The study also reported the highest breast cancer mortality rates were from African Americans, patients with low health care resource availability zip-codes, and low socio-economic status zip-codes [5].

In California, incidence rates and mortality rates due to breast cancer decreased in 2016 , by $8 \%$ and $36 \%$, respectively [24]. In 2013, California had 25,632 new cases of breast cancer with 4,361 breast cancer-attributable deaths [24]. According to a 2011 study by the California Department Of Health Care Services (CDHCS), the age-adjusted rate of breast cancer in women was 122 per 100,000 , with the highest incidence rate in White women $(123$ per 100,000), the second highest rate in Black women (121 per 100,000), and the lowest in Hispanic women (92 per 100,000) 
[25]. The highest mortality rates were in Black women (32 per $100,000)$, despite having a lower incidence than White women [25]. In 2012, there was a higher rate of screening in women who were nationally insured (76\%)than in women who had Medicaid (52\%) [25]. Lower socioeconomic status could be an indicator of risk for women when it comes to breast cancer. The CDHCS also showed that in 2012 Asian/Pacific Islander women were the most likely to have undergone mammography screening in the last two years 54\%, followed by Latino women $52 \%$, White women $44 \%$, Black women $39 \%$, and finally American Indian/Alaska Native women $37 \%$ [25]. Understanding state-specific screening rates allows policy makers to address disparities in each state.

Both Michigan and California have resources for people with a low socioeconomic status and for those who are uninsured. The Breast Cancer And Cervical Cancer Control Navigation Program (BCCCNP) in Michigan provides free mammography screenings for women ages 40 to 69 who have a low income and are uninsured [26]. In California, the Every Woman Counts (EWC) program provides free breast cancer screenings to lowincome women as well as educational and preventive services in the community [25]. Further studies could determine why the majority of women who do not participate in mammography screenings are most commonly eligible for such programs. Many women who are uninsured or on a low coverage service such as Medicaid have co morbidities that severely lower their survival time and chance for a full recovery [27]. However, opportunities for free quality care for uninsured, low income women drastically decrease post-diagnosis [27]. Limitations in accessibility of care could be contributing factors in racial disparities. The purpose of this study is to determine if there exists a significant difference between various racial and ethnic groups in Michigan and California. This study focuses on White Hispanic, White non-Hispanic, Black Hispanic, and Black non-Hispanic breast cancer patients in Michigan and California from 1973-2012 to statistically analyze variations in survival duration and age at diagnosis.

\section{Materials and Methods}

Breast cancer patient data was collected from the Surveillance, Epidemiology, and End Results (SEER) database for two states, Michigan and California. Four ethnic populations were considered: White Hispanics, White non-Hispanics, Black Hispanics, and Black non-Hispanics. There were 121,358 patients available for Michigan and 104,733 patients available for California in the SEER database. Michigan had a sample size of 3,078 patients, with sample sizes of 1,000 for White Hispanics, White non-Hispanics, and Black non-Hispanics. Black Hispanics had a smaller sample size of 78 patients. The sample size for California was 3,133 patients with sample sizes of 1,000 for White Hispanics, White non-Hispanics, and Black non-Hispanics. Black Hispanics had a smaller sample size of 133 patients. The small sample size for Black Hispanics in each state was due to limited available data from the SEER database.

Figure 1 outlines the distribution of sample size for this study according to the separation of each group from the available patient data $(\mathrm{N}=226,091)$. Available patient data for Michigan was 121,358 and available patient data for California was 104,733. Sample sizes were taken for the four ethnic groups: White Hispanics, White non-Hispanics, Black Hispanics, and Black non-Hispanics. Total sample sizes for each state were 3,078 for Michigan and 3,133 for California.

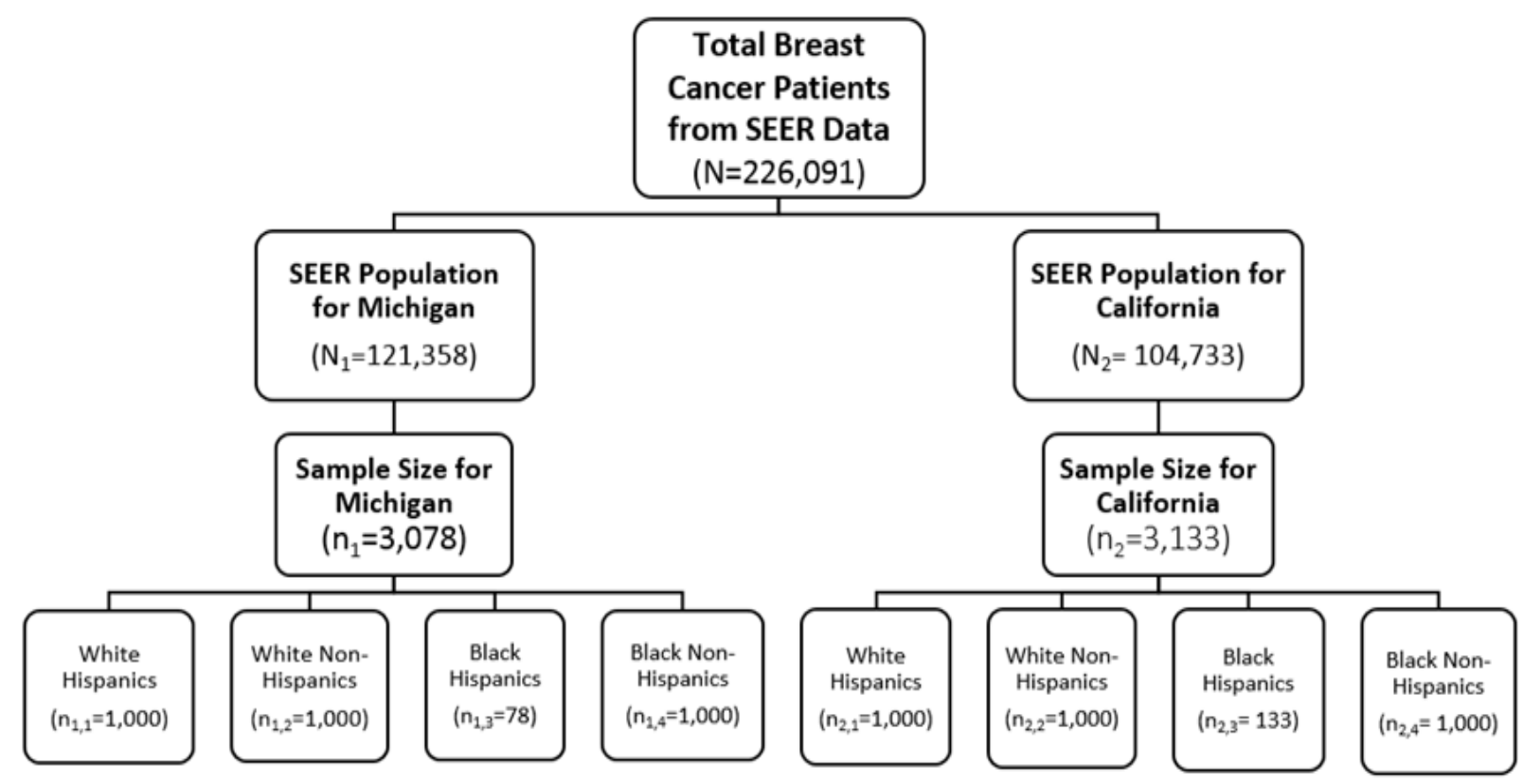

Figure 1: Selection of Sample Sizes for White Hispanics, White Non-Hispanics, Black Hispanics, and Black Non-Hispanics for California and Michigan 
A summary of statistics was calculated for age of diagnosis (years) and survival duration (months) for each ethnic population in Michigan and California. The Cox Proportion Hazard Ratio function and goodness-of-fit assessments for numerous probability distributions were used to compare the survival between each race and ethnic group. A cross tabulation analysis was performed between categories of age of diagnosis, and race and ethnicity. Probability distributions were analyzed for each ethnic group for both California and Michigan. The SPSS statistical software (version 23.0), Easy fit distribution fitting software (version 5.5), and descriptive and inferential statistics were used to analyze the data $[28,29]$.

\section{Results and Discussion}

This study investigated the differences in age of diagnosis and mean survival time between race and ethnicity for breast cancer patients. The mean age of diagnosis for White Hispanics, White non-Hispanics, Black Hispanics, and Black non-Hispanics are listed for each state in Table1. In Michigan, the lowest mean age of diagnosis was found in White Hispanics at 56.9 years $(S D=13.9$ ). Black Hispanics in Michigan had a mean age of diagnosis slightly higher than the lowest group, at 57.1 years ( $S D=14.5)$, followed by Black non-Hispanics at 58.5 years (SD =14.1). The highest mean age of survival for Michigan was found in White nonHispanics at 62.2 years (SD $=14.0$ ). In California, Black Hispanics had the lowest mean age of diagnosis at 55.5 years $(S D=14.9)$. The next highest mean age of diagnosis was in White Hispanics at 59.2 years (SD $=14.6)$, followed by Black non-Hispanics at 59.2 years $(\mathrm{SD}=14.4)$. White non-Hispanics also had the highest mean age of diagnosis in California at 62.8 years $(\mathrm{SD}=14.1)$. Independent samples t-tests were performed at a $5 \%$ level of significance and it was found that White Hispanics in California had a significantly higher mean age of diagnosis than White Hispanics in Michigan $(p<0.001)$.

The mean survival times for each ethnic group are also listed for the two states along with the mean age of diagnosis in Table 1. The lowest survival time found for Michigan was for Black Hispanics at 78.1 months (6.5 years; SD = 62.2 months), followed by Black non-Hispanics at 93.7 months (7.8 years; SD $=89.1$ months), and White Hispanics at 101.0 months (8.4 months; SD = 89.5 months). The highest survival time in Michigan was in White non-Hispanics at 111.5 months (9.3 years; $\mathrm{SD}=94.2$ months). In California, Black non-Hispanics showed the lowest mean survival time at 102.9 months (8.6 years; SD $=93.0$ months), followed by White Hispanics at 107.9 months (9.0 years; SD $=95.2$ months), and Black Hispanics at 110.7 months (9.2 years; SD $=100.3$ months). White non-Hispanics in California had the highest mean survival time at 125.8 months (10.5 years; SD $=102.1$ months). Independent samples t-tests were performed at a $5 \%$ level of significance and it was found that White non-Hispanics, Black Hispanics, and Black non-Hispanics in California had significantly higher mean survival times compared with Michigan $(p<0.05)$.

\begin{tabular}{|c|c|c|c|c|c|}
\hline \multirow[t]{2}{*}{ State } & \multirow[t]{2}{*}{ Race and Ethnicity } & \multicolumn{2}{|c|}{ Age of Diagnosis (yrs) } & \multicolumn{2}{|c|}{ Survival Time (months) } \\
\hline & & Mean & Std Dev* & Mean & Std Dev* \\
\hline \multirow[t]{4}{*}{ Michigan } & White Hispanic & 56.9 & 13.9 & 101.0 & 89.5 \\
\hline & White non-Hispanic & 62.2 & 14.0 & 111.5 & 94.2 \\
\hline & Black Hispanic & 57.1 & 14.5 & 78.1 & 62.2 \\
\hline & Black non-Hispanic & 58.5 & 14.1 & 93.7 & 89.1 \\
\hline \multirow[t]{4}{*}{ California } & White Hispanic & 59.2 & 14.6 & 107.9 & 95.2 \\
\hline & White non-Hispanic & 62.8 & 14.1 & 125.8 & 102.1 \\
\hline & Black Hispanic & 55.5 & 14.9 & 110.7 & 100.3 \\
\hline & Black non-Hispanic & 59.2 & 14.4 & 102.9 & 93.0 \\
\hline
\end{tabular}

\section{* Std Dev= standard deviation.}

Independent sample t-test revealed that White Hispanics in California had a significantly higher mean age of diagnosis than White Hispanics in Michigan $(p<0.001)$.

A comparison between each state for the mean ages of diagnosis is shown in Figure 2. This figure shows that there are not many differences between California and Michigan on the mean age of diagnosis except there was a difference between the mean ages of diagnosis for White Hispanics. White Hispanics in California had a significantly higher mean age of diagnosis than White Hispanics in Michigan $(p<0.001)$. The lack of statistical significance for Black Hispanics could be due to small sample size in each state.

Figure 3 shows the comparison between Michigan and
California for mean survival times. California showed consistently higher mean survival times compared to Michigan. At a significance level of $\alpha=0.05$, White non-Hispanics ( $p=0.001$ ), Black Hispanics $(p=0.004)$, and Black non-Hispanics $(p=0.023)$ in California had significantly higher survival times than Michigan.

Table 2 describes the distribution of breast cancer patients from the population available in the SEER database for each state. A Chi-squared analysis of the frequencies of ethnicities between Michigan and California was significant at $\alpha=0.01$ level $(p<0.001)$. 


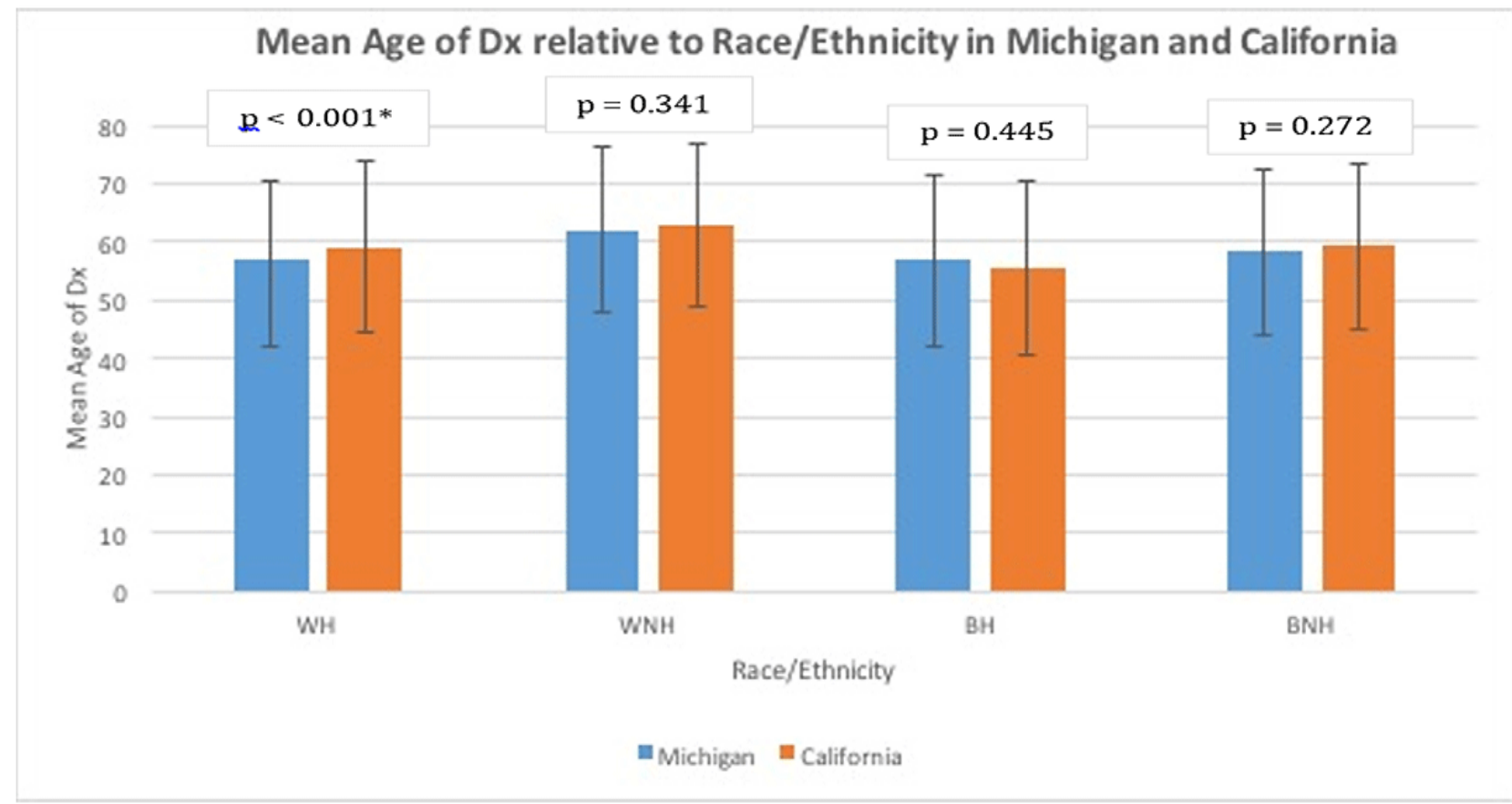

Figure 2: Mean ages of diagnosis for race/ethnicity in Michigan and California

The graph shows a visual comparison between Michigan and California for White Hispanics, White Non-Hispanics, Black Hispanics, and Black Non-Hispanics. Michigan is represented in blue and California is represented in orange. ${ }^{*}$ The mean age of diagnosis of White Hispanics in California is significantly higher than White Hispanics in Michigan $(p<0.001)$. None of the other ethnic groups had a significant difference between each state. Abbreviations: WH=White Hispanics; WNH=White Non-Hispanics; BH=Black Hispanics; BNH=Black Non-Hispanic.

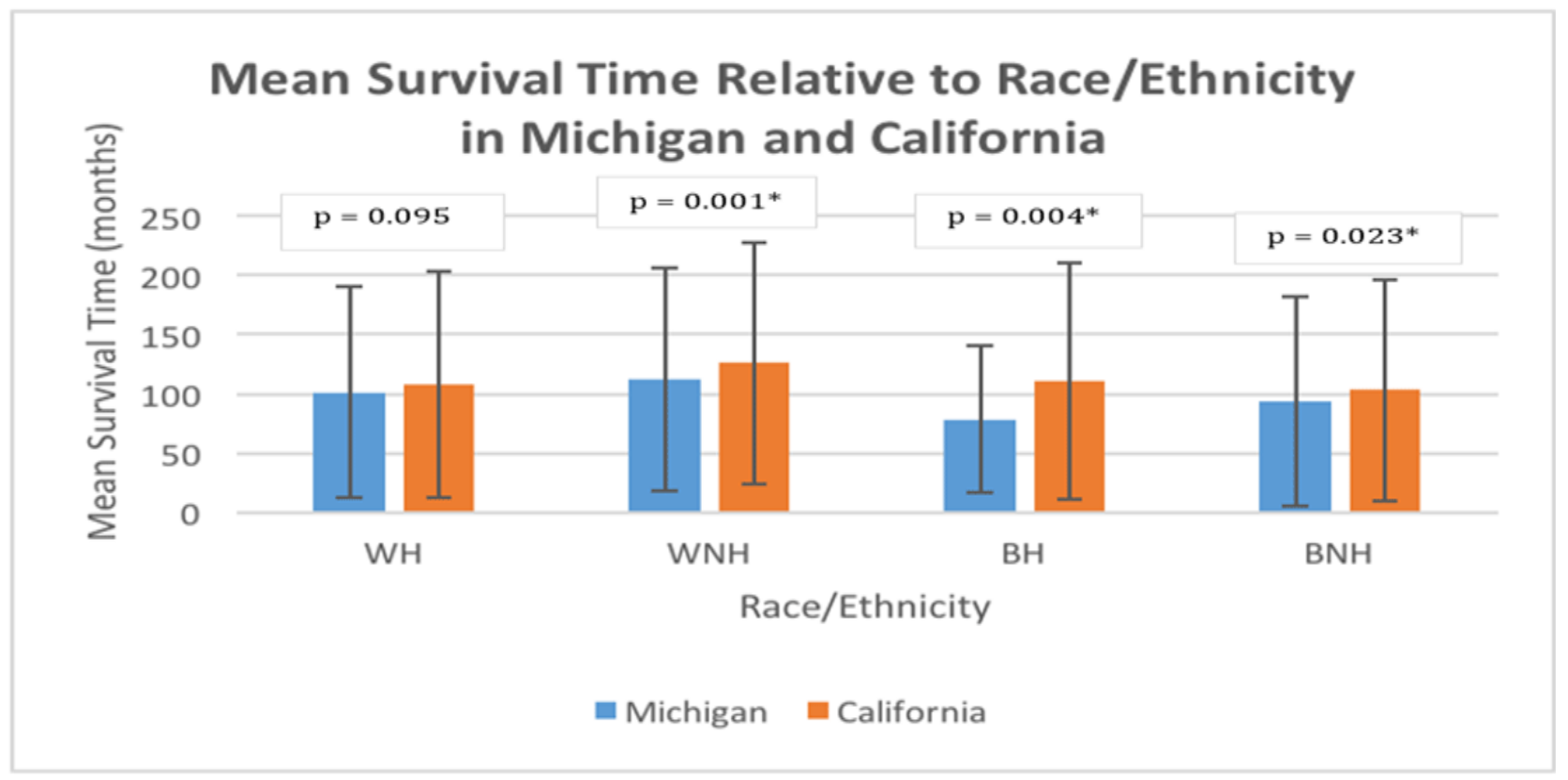

Figure 3: Mean survival times for race/ethnicity in Michigan and California

The graph shows a visual comparison between Michigan and California for White Hispanics, White Non-Hispanics, Black Hispanics, and Black Non-Hispanics. Michigan is represented in blue and California is represented in orange. California showed consistently higher mean survival times among all four ethnic groups. *White non-Hispanics, Black Hispanics, and Black non-Hispanics in California had significantly higher mean survival times than the same three ethnic groups in Michigan $(p<0.05)$. 
Table 2: Distribution of ethnic patients between Michigan and California

\begin{tabular}{|l|c|c|c|c|c|}
\hline & \multicolumn{2}{|c|}{ Michigan } & \multicolumn{2}{c}{ California } & Significance \\
\hline White Hispanics & Frequency & Percent (\%) & Frequency & Percent (\%) \\
\hline White Non-Hispanics & 1,502 & 1.24 & 8,630 & 8.24 \\
& 95,877 & 79.00 & 85,837 & $0.001^{*}$ \\
\hline Black Hispanics & 78 & & & 0.13 \\
\hline Black Non-Hispanics & 23,901 & 0.06 & 133 & 9.68 \\
\hline
\end{tabular}

The distribution of ethnicity for both Michigan and California from the available population in the SEER database. Chi-squared distribution of the relationship between ethnic groups and state were found to be statistically significant $(p<0.001)$.

Table 3 shows a cross tabulation analysis describing the relationship between age at diagnosis, and race and ethnicity in California and Michigan. Age of diagnosis is split into four different categories including, ages less than 30 , ages 30 to 49 years, ages 50 to 69 years and ages that fall at or above 70 years. Percentages of frequencies were taken as a part of the total number of patients in each age category. In Michigan, the highest percentage of patients for the less than 30 age group was White Hispanics (44.8\%), for the 30-49 age groups it was White Hispanics (38.6\%), for the 5069 age group, Black non-Hispanics (33.2\%), and for the greater than 70 age group, White non-Hispanics (41.7\%). In California, the highest percentage of patients for the less than 30 age group was Black non-Hispanics (40.9\%), for the 30-49 age groups it was White Hispanics (35.8\%), for the 50-69 age group, Black nonHispanics (32.9\%), and for the greater than 70 age group, White non-Hispanics (39.1\%).
Figure 4 shows the survival curve for the state of Michigan separated by race and ethnicity. According to this survival curve, Black Hispanics and Black non-Hispanics have the lowest survival times compared to White Hispanics and White non-Hispanics for Michigan. However, the survival times for White Hispanics appear to be similar to Black Hispanics and Black non-Hispanics. White Hispanics show a consistently higher survival rate compared to Black Hispanics, Black non-Hispanics, and White non-Hispanics.

Figure 5 shows the survival curve for the state of California for Black Hispanics, Black non-Hispanics, White Hispanics, and White non-Hispanics. As can be seen in the figure, Black nonHispanics consistently have the lowest survival time compared to the other three ethnic groups. White Hispanics and White nonHispanics were very similar in the shape of their survival curves. The shape of the survival curve for Black Hispanics changes in the middle of the curve.

Table 3: Cross tabulation analysis of age category compared to racial/ethnic group for Michigan and California

\begin{tabular}{|c|c|c|c|c|c|c|c|c|c|}
\hline \multicolumn{2}{|c|}{ Age Groups } & \multicolumn{4}{|c|}{ Michigan } & \multicolumn{4}{|c|}{ California } \\
\hline \multicolumn{2}{|r|}{ BH } & BNH & WH & WNH & BH & BNH & WH & WNH & \\
\hline \multirow[t]{2}{*}{$<30$} & Count & 0 & 9 & 13 & 7 & 2 & 9 & 6 & 5 \\
\hline & $\%$ within & $0.0 \%$ & $31.0 \%$ & $44.8 \%$ & $24.1 \%$ & $9.1 \%$ & $40.9 \%$ & $27.3 \%$ & $22.7 \%$ \\
\hline \multirow[t]{2}{*}{$30-49$} & Count & 28 & 287 & 327 & 206 & 55 & 257 & 279 & 189 \\
\hline & $\%$ within & $3.3 \%$ & $33.8 \%$ & $38.6 \%$ & $24.3 \%$ & $7.1 \%$ & $32.9 \%$ & $35.8 \%$ & $24.2 \%$ \\
\hline \multirow[t]{2}{*}{$50-69$} & Count & 32 & 466 & 450 & 454 & 50 & 483 & 466 & 468 \\
\hline & $\%$ within & $2.3 \%$ & $33.2 \%$ & $32.1 \%$ & $32.4 \%$ & $3.4 \%$ & $32.9 \%$ & $31.8 \%$ & $31.9 \%$ \\
\hline \multirow[t]{2}{*}{$>70$} & Count & 18 & 238 & 210 & 333 & 26 & 251 & 249 & 338 \\
\hline & $\%$ within & $2.3 \%$ & $29.8 \%$ & $26.3 \%$ & $41.7 \%$ & $3.0 \%$ & $29.1 \%$ & $28.8 \%$ & $39.1 \%$ \\
\hline \multirow[t]{2}{*}{ Total } & Count & 78 & 1000 & 1000 & 1000 & 133 & 1000 & 1000 & 1000 \\
\hline & \% within & $2.5 \%$ & $32.5 \%$ & $32.5 \%$ & $32.5 \%$ & $4.2 \%$ & $31.9 \%$ & $31.9 \%$ & $31.9 \%$ \\
\hline
\end{tabular}

Percentages were taken out of the total number of patients for each age category per state. Percentages for each age category add to a total of $100 \%$. A Chi-squared analysis of Age groups vs. Michigan and Age groups vs. California were statistically significant $(p<0.001)$. 


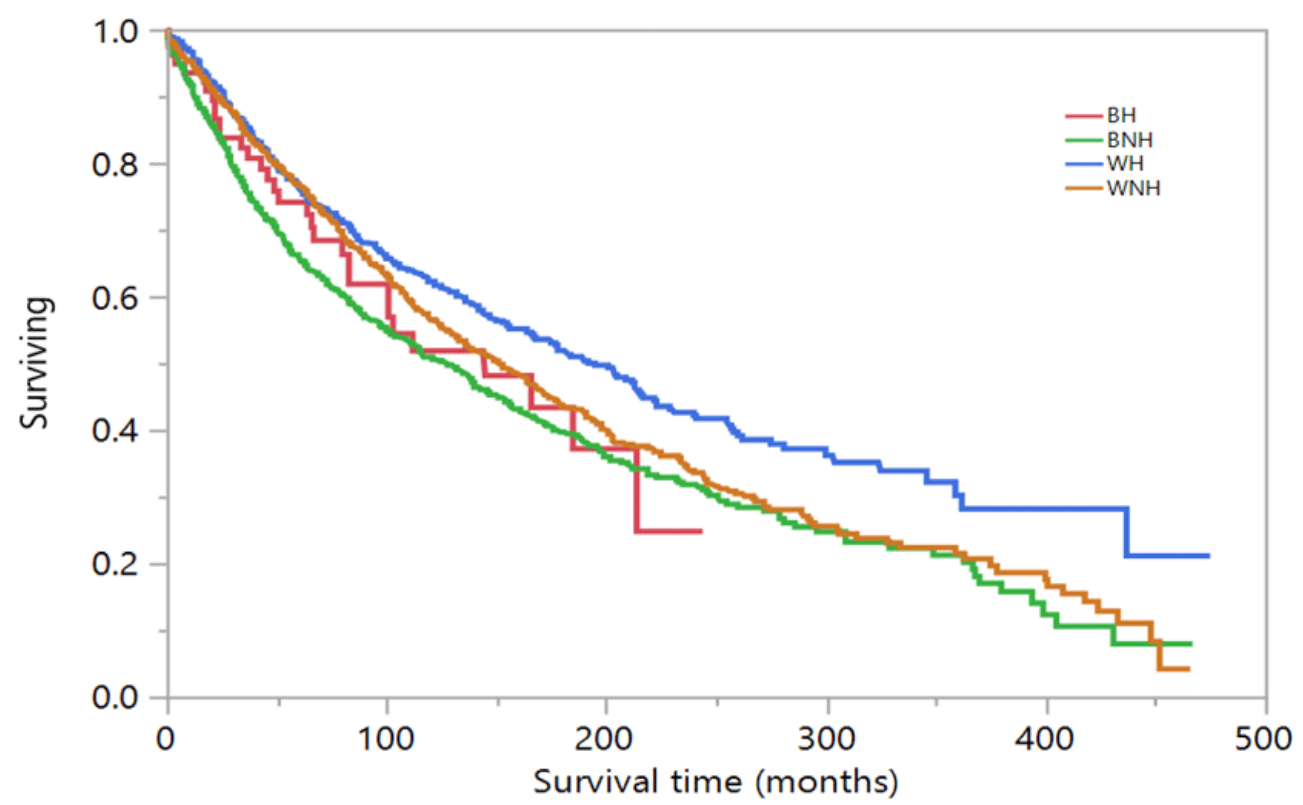

Figure 4: Survival curve for Michigan with race and ethnicity

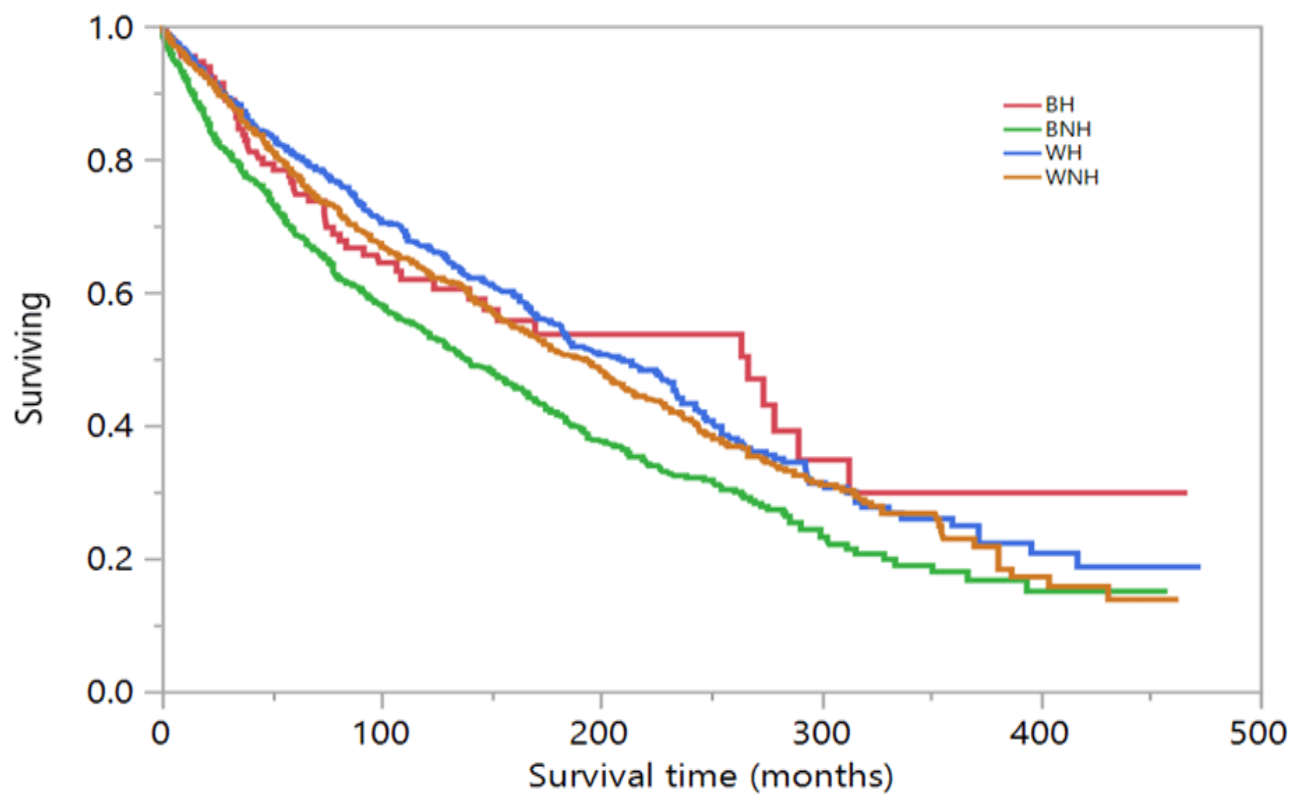

Figure 5: Survival curve for California with race and ethnicity

Table 4 shows the Cox Proportion Hazard ratios for survival times of breast cancer patients in Michigan and California. In Michigan, with Black non-Hispanics as the reference group, Black Hispanics had the highest hazard ratio at $1.014(95 \%$ CI: 0.709 , 1.449), followed by White non-Hispanics at 0.726 (95\% CI: 0.642 , 0.821), and White Hispanics at 0.716 (95\% CI: 0.627, 0.817). The hazard ratios for White non-Hispanics and White Hispanics were statistically significant at $\alpha=0.01$ level, showing higher survival than the reference group, Black non-Hispanic. In California, with Black non-Hispanics as the reference group, Black Hispanics had the highest hazard ratio value at 0.799 (95\% CI: 0.602, 1.061), followed by White non-Hispanics at 0.681 (95\% CI: 0.601, 0.770), and White Hispanics at 0.671 (95\% CI: 0.588, 0.766). The hazard ratios for White non-Hispanics and White Hispanics were also statistically significant at $\alpha=0.01$ level, showing higher survival than the reference group, Black non-Hispanic. 
Table 4: Cox proportional hazard ratios for breast cancer patients' mean survival time for Michigan and California

Cox Proportional Hazard Ratios for Michigan

\begin{tabular}{|c|c|c|c|c|c|c|c|c|}
\hline \multicolumn{9}{|c|}{ 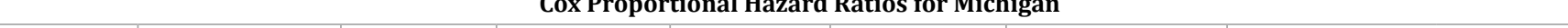 } \\
\hline & \multirow[b]{2}{*}{ B } & \multirow[b]{2}{*}{ SE } & \multirow[b]{2}{*}{ Wald } & \multirow[b]{2}{*}{ D.F. } & \multirow[b]{2}{*}{ Sig. } & \multirow{2}{*}{$\begin{array}{c}\text { Hazard Ratios } \\
\text { (HR) }\end{array}$} & \multicolumn{2}{|c|}{ 95\% CI for HR } \\
\hline & & & & & & & Lower & Upper \\
\hline BNH & Ref. & & & & & & & \\
\hline BH & 0.014 & 0.182 & 0.006 & 1 & 0.940 & 1.014 & 0.709 & 1.449 \\
\hline WH & -0.334 & 0.067 & 24.596 & 1 & $<0.001^{*}$ & 0.716 & 0.627 & 0.817 \\
\hline WNH & -0.321 & 0.063 & 26.075 & 1 & $<0.001^{*}$ & 0.726 & 0.642 & 0.821 \\
\hline \multicolumn{9}{|c|}{ Cox Proportional Hazard Ratios for California } \\
\hline & & & & & & Hazard Ratios & \multicolumn{2}{|c|}{ 95\% CI for HR } \\
\hline & B & SE & Wald & D.F. & Sig. & (HR) & Lower & Upper \\
\hline BNH & Ref. & & & & & & & \\
\hline BH & -0.224 & 0.144 & 2.410 & 1 & 0.121 & 0.799 & 0.602 & 1.061 \\
\hline WH & -0.399 & 0.067 & 35.013 & 1 & $<0.001^{*}$ & 0.671 & 0.588 & 0.766 \\
\hline WNH & -0.385 & 0.063 & 37.275 & 1 & $<0.001 *$ & 0.681 & 0.601 & 0.770 \\
\hline
\end{tabular}

Table 5 shows a goodness-of-fit assessment for the survival probabilities for each ethnic subgroup in California and Michigan. There were two primary distributions that were followed by the different sub-populations: t-distribution, and Chi-squared distribution. For Michigan the highest Chi-squared value for those groups that followed t-distribution was in White Hispanics. Black Hispanics were the only group that followed the chi-squared in Michigan. In California among those groups that followed the t-distribution, White Hispanics contributed to the highest Chisquared value. Similar to Michigan, Black Hispanics were the only group in California that followed the Chi-squared distribution.

\section{Conclusions}

This study considers two states showing the differences in age of diagnosis and survival times for breast cancer patients among different racial and ethnic groups. The mean age of diagnosis was almost similar between Michigan and California. In Michigan, the difference between the highest and lowest mean age of diagnosis for White non-Hispanics and White Hispanics was 5.3 years. In California, the difference between the highest and lowest mean age of diagnosis for White non-Hispanics and Black Hispanics was slightly higher at 7.3 years. These disparities may be due to genetic differences in the population, socioeconomic differences, or differences in health care policies between each state.

Table 5: Goodness-of-fit assessment of survival probability among breast cancer patients in Michigan and California

\begin{tabular}{|c|c|c|c|c|c|}
\hline State & $\begin{array}{c}\text { Race/ } \\
\text { Ethnicity }\end{array}$ & $\begin{array}{l}\text { Probability } \\
\text { Distribution }\end{array}$ & Kolmogorov-Smirnov & Anderson-Darling & Chi-Squared \\
\hline \multirow[t]{4}{*}{ Michigan } & White Hispanic & t-distribution & 0.93804 & 6548.8 & 17819 \\
\hline & White non-Hispanic & t-distribution & 0.9331 & 6706.6 & 17628 \\
\hline & Black Hispanic & $\begin{array}{l}\text { Chi-squared } \\
\text { distribution }\end{array}$ & 0.44806 & 315.36 & 97 \\
\hline & Black non-Hispanic & t-distribution & 0.92902 & 6178.8 & 17587 \\
\hline \multirow[t]{4}{*}{ California } & White Hispanic & t-distribution & 0.93306 & 6578.9 & 17570 \\
\hline & White non-Hispanic & t-distribution & 0.94988 & 7124.1 & 3848.9 \\
\hline & Black Hispanic & $\begin{array}{l}\text { Chi-squared } \\
\text { distribution }\end{array}$ & 0.50295 & 774.21 & 249.44 \\
\hline & Black non-Hispanic & $\mathrm{t}$-distribution & 0.91774 & 6265.4 & 17129 \\
\hline
\end{tabular}


The differences between states for mean survival times were more consistent than the differences between the mean ages of diagnosis. California showed consistently higher survival times across all four ethnic groups. This is also observed in the individual survival curves for each state. The survival curve for California has less apparent disparities between the highest and lowest groups than in the survival curve in Michigan. Black Hispanics in Michigan had the lowest mean survival time of 78.1 months ( $S D=62.2$ ), while Black Hispanics in California had a mean survival time of 110.7 months ( $\mathrm{SD}=100.3)$. In Michigan, White non-Hispanics (HR $=0.726 ; 95 \% \mathrm{CI}=[0.642-0.821])$ and White Hispanics (HR=0.716; 95\% CI=[0.627-0.817]) both showed a significant difference in mean survival time to the reference group, Black non-Hispanics. In California, White non-Hispanics $(\mathrm{HR}=0.681 ; 95 \% \mathrm{CI}=[0.601-0.770])$, and White Hispanics (HR $=0.671 ; 95 \% \mathrm{CI}=[0.588-0.766])$ showed a significant difference in mean survival time with the reference group, Black nonHispanics.

Limitations to this study include the availability of demographic and socioeconomic data. SEER is a reliable source for cancer data but lacks some specific variables and characteristics that could provide information for physicians and public health officials to tailor prevention and intervention methods to specific populations. This analysis of breast cancer patient survival includes only two states. Michigan and California were chosen to represent two separate regions of the U.S., but only considering two states also limits the scope of this study. Another limitation of this study includes the small sample sizes for the Black Hispanics group in both states. Due to a lack of available data, this group had a much smaller sample size compared to the other three ethnic groups. This could affect the apparent impact of breast cancer on this subgroup and it is important that breast cancer in this ethnic group is explored further.

The results show there is a significant difference between the mean survival times, and therefore, we can assume that these different demographics have different factors affecting breast cancer rates in their populations. Further studies should analyze these differences to help tailor preventive programs for each of these populations. This could cause incidence rates to decline and no longer have significant disparities between various racial and ethnic populations.

\section{Acknowledgement}

This research was funded by the Laura W. Bush Institute for Women's Health at TTUHSC (Grant\#HD181605). The authors are grateful to the National Cancer Institute in the United States for giving them access to the SEER's database.

\section{References}

1. Samson ME, Porter NG, Hurley DM, Adams SA, Eberth JM. Disparities in Breast Cancer Incidence, Mortality, and Quality of Care among African American and European American Women in South Carolina. South Med J. 2016;109(1):24-30. doi: 10.14423/SMJ.0000000000000396
2. Graham A colditz. Breast Cancer Epidemiology and Risk Factors: Epidemiology, Reproductive Factors, Endogenous Sex Hormones and Risk of Breast Cancer. 2015.

3. U.S. breast cancer statistics. Breast Cancer.

4. The costs of breast cancer in the U.S.

5. Harper S, Lynch J, Meersman SC, Breen N, Davis WW, Reichman MC. Trends in area-socioeconomic and race-ethnic disparities in breast cancer incidence, stage at diagnosis, screening, mortality, and survival among women ages 50 years and over (1987-2005). Cancer Epidemiol Biomarkers Prev. 2009;18(1):121-131. doi: 10.1158/1055-9965.EPI08-0679

6. Li CI, Malone KE, Daling JR. Differences in breast cancer hormone receptor status and histology by race and ethnicity among women 50 years of age and older. Cancer Epidemiol Biomarkers Prev. 2002;11(7):601-607.

7. Clarke CA, Keegan TH, Yang J, Press DJ, Kurian AW, Patel AH, Lacey JV Jr. et al. Age-specific incidence of breast cancer subtypes: understanding the black-white crossover. J Natl Cancer Inst. 2012;104(14):10941101. doi: $10.1093 /$ jnci/djs264

8. Buzdar AU. Endocrine therapy in the treatment of metastatic breast cancer. Semin Oncol. 2001;28(3): 291-304.

9. Arpino G, Weiss H, Lee AV, Schiff R, De Placido S, Osborne CK, Elledge RM, et al. Estrogen Receptor-Positive, Progesterone ReceptorNegative Breast Cancer: Association With Growth Factor Receptor Expression and Tamoxifen Resistance. JNCI J Natl Cancer Inst. 2005;97(17): 1254-1261. doi: 10.1093/jnci/dji249

10. Mitri Z, Constantine T, O'Regan R. The HER2 Receptor in Breast Cancer: Pathophysiology, Clinical Use, and New Advances in Therapy. Chemother Res Pract. 2012;2012:743193. doi: 10.1155/2012/743193

11. Cancer of the Breast (Female) - SEER Stat Fact Sheets.

12.Schneider EC, Zaslavsky AM, Epstein AM. Racial disparities in quality of care for enrollees in medicare managed care. JAMA. 2002; 287(10):1288-1294

13. Hunter CP. Epidemiology, stage at diagnosis, and tumor biology of breast carcinoma in multiracial and multiethnic populations. Cancer. 2000;88(5 suppl):1193-1202.

14. McCarthy EP, Burns RB, Coughlin SS, Freund KM, Rice J, Marwill SL, et al. Mammography use helps to explain differences in breast cancer stage at diagnosis between older black women and white women. Ann Intern Med. 1998;128(9) :729-736.

15. Tejeda S, Thompson B, Coronado GD, Martin DP. Barriers and facilitators related to mammography use among lower educated Mexican women in the USA. Soc Sci Med. 2009;68(5):832-839. doi: 10.1016/j.socscimed.2008.12.023

16. Cui Y, Whiteman MK, Langenberg $\mathrm{P}$, Sexton M, Tkaczuk KH, Flaws JA, et al. Can obesity explain the racial difference in stage of breast cancer at diagnosis between black and white women? J Womens Health Gend Based Med. 2002;11(6) :527 -536. doi: $10.1089 / 152460902760277886$

17.USPSTF, AAFP issue final breast cancer screening recommendations. 2016.

18. Qin X, Tangka F, Guy Jr G, Howard D. Mammography rates after the 2009 revision to the united states preventive services task force breast cancer screening recommendation. Cancer Causes \& Control. 2016;28(1):41-48. doi: 10.1007/s10552-016-0835-1 
19. Khan HMR, Ibrahimou B, Gabbidon K, Abdool-ghany F, Ullah D, Stewart TS, et. al.Statistical Estimates from Black Non-Hispanic Female Breast Cancer Data. Asian Pac J Cancer Prev. 2014;15(19):8371-8376.

20. Khan HM, Saxena A, Gabbidon K, Ross E, Shrestha A. Statistical applications for the prediction of white Hispanic breast cancer survival. Asian Pac J Cancer Prev. 2014;15(14):5571-5575.

21. Khan HM, Saxena A, Gabbidon K, Stewart TS, Bhatt C. Survival analysis for white non-Hispanic female breast cancer patients. Asian Pac J Cancer Prev. 2014;15(9): 4049-4054.

22. Khan HM, Saxena A, Vera V, Gabbidon K, Perea N, Shauna-jeanne T, et. al. Black Hispanic and Black Non-Hispanic Breast Cancer Survival Data Analysis with Half-normal Model Application. Asian Pac J Cancer Prev. 2014;15(21):9453-9458.

23. Meliker JR, Goovaerts P, Jacquez GM, Avruskin GA, Copeland G. Breast and prostate cancer survival in Michigan: can geographic analyses assist in understanding racial disparities? Cancer. 2009;115(10):22122221. doi: 10.1002/cncr.24251
24. American Cancer Society. California cancer facts \&amp; figures. California Department of Public Health. 2016.

25. Health disparities in the medi-cal population: Mammography screening. California Department of Health Care Services.

26. Breast cancer in Michigan. Michigan Department of Health \& Human Services. 2016. USPSTF, AAFP issue final breast cancer screening recommendations.

27. Bradley CJ, Given CW, Roberts C. Race, socioeconomic status, and breast cancer treatment and survival. J Natl Cancer Inst. 2002;94(7):490-496

28. SPSS. 2015; Statistical package for the social sciences (SPSS), IBM Inc.

29. Easy Fit. Distribution fitting made easy, math wave-data analysis \& simulation, version 5.5. 2015. 